\title{
Erlotinib with pemetrexed/cisplatin for patients with EGFR wild-type lung adenocarcinoma with brain metastases
}

\author{
YALEI ZHANG ${ }^{1,2^{*}}$, HAIHONG YANG ${ }^{1,2^{*}}$, XINYUN YANG ${ }^{2,3}$, QIUHUA DENG ${ }^{2,4}$, \\ MEILIN ZHAO ${ }^{1,2}, \mathrm{XIN} \mathrm{XU}^{1,2}$ and JIANXING HE $\mathrm{HE}^{1,2}$ \\ ${ }^{1}$ Department of Cardiothoracic Surgery, ${ }^{2}$ State Key Laboratory of Respiratory Diseases, ${ }^{3}$ Department of Pharmacy; \\ ${ }^{4}$ Center for Translational Medicine, The First Affiliated Hospital of Guangzhou Medical University, \\ Guangzhou, Guangdong 510120, P.R. China
}

Received November 4, 2013; Accepted January 22, 2014

DOI: $10.3892 / \mathrm{mco} .2014 .256$

\begin{abstract}
Erlotinib and pemetrexed have been approved for the second-line treatment of non-small cell lung cancer. Recent reports indicated that erlotinib and pemetrexed exerted synergistic effects against lung adenocarcinoma. The available treatment options for lung cancer with brain metastases (BM) are currently limited. In the present study, we investigated the efficacy of the combined administration of erlotinib and pemetrexed in 9 patients with epidermal growth factor receptor $(E G F R)$ wild-type lung adenocarcinoma with BM. Pemetrexed $\left(500 \mathrm{mg} / \mathrm{m}^{2}\right)$ and cisplatin $\left(20 \mathrm{mg} / \mathrm{m}^{2}\right)$ were administered on day 1 and days 1-3, respectively. Erlotinib (150 mg) was administered daily on days 4-20. The 9 patients harbored EGFR wild-type mutation in the primary tumor tissues. With regard to the BM, no patients achieved complete remission, 7 patients exhibited a partial response (PR), 1 had stable disease (SD) and 1 had progressive disease (PD). As regards the extracranial tumors, 3 patients exhibited a PR, 2 had SD, 3 had PD and 1 was not applicable. The performance status and the symptoms improved in 3 patients following treatment. The median progression-free survival for intracranial and extracranial disease control was 179 and 146.5 days, respectively. The median overall survival was 197.4 days. Therefore, erlotinib combined with pemetrexed/cisplatin, was found to be effective in the treatment of patients with EGFR wild-type lung adenocarcinoma.
\end{abstract}

Correspondence to: Dr Jianxing He, Department of Cardiothoracic Surgery, The First Affiliated Hospital of Guangzhou Medical University, 151 Yanjiang Road, Guangzhou, Guangdong 510120, P.R. China

E-mail: hejx@vip.163.com

${ }^{*}$ Contributed equally

Key words: erlotinib, pemetrexed, adenocarcinoma, brain metastases

\section{Introduction}

Non-small cell lung cancer (NSCLC) is the leading cause of cancer-related mortality worldwide. Brain metastases (BM) commonly occur in patients with lung adenocarcinoma and are usually associated with a poor prognosis and poor quality of life for the patients. A total of 30-50\% of NSCLC patients develop BM prior to or during treatment (1). The currently available treatment options for patients with BM are limited. Generally, the prognosis of BM is poor. The median survival with standard treatment is 2 months, which involves expensive and invasive procedures, such as systemic treatment with corticosteroids and whole-brain radiotherapy, with or without stereotactic radiosurgery (2). Thus, it is imperative to develop additional effective therapies for patients with BM.

Erlotinib and pemetrexed were reported to be effective in lung adenocarcinoma patients with BM. Erlotinib, as an epidermal growth factor receptor $(E G F R)$ tyrosine-kinase inhibitor (TKI), is a small molecular agent that is able to cross the blood-brain barrier. Erlotinib was reported to significantly improve the response rate of metastatic brain tumors and survival in lung adenocarcinoma patients with asymptomatic synchronous BM, particularly those with EGFR-activating mutations in exon 19 or $21(3,4)$. However, EGFR wild-type patients, compared to mutant patients, exhibited significantly poorer BM treatment responses and a shorter survival after BM diagnosis (5). Pemetrexed is an inhibitor of thymidylate synthase (TS), dihydrofolate reductase and glycinamide ribonucleotide formyltransferase (6). It was recently reported that the combination of pemetrexed and platinum may be particularly effective in terms of intracranial radiological response and overall survival (OS) in NSCLC patients with newly diagnosed BM (7). Resistance to pemetrexed may be attributed mainly to an increased TS expression (8).

The heterogeneity of NSCLC tumors provides a strong rationale for using combination therapy with targeted agents that have different mechanisms of action. Moreover, different combinations may exert synergistic effects (9). In a previous study, erlotinib in combination with pemetrexed for treating patients with advanced NSCLC was found to be well-tolerated and exhibited a promising efficacy in a phase I dose-finding study (10). In small samples, this combination regimen also 
exhibited promising efficacy in pretreated advanced lung adenocarcinoma (11).

To build on these previously reported clinical data, we hypothesized that erlotinib in combination with pemetrexed and cisplatin may be more effective for the treatment of patients with $E G F R$ wild-type lung adenocarcinoma with BM.

\section{Patients and methods}

Patients. A total of 9 lung adenocarcinoma patients were diagnosed and treated with a combination of erlotinib and pemetrexed/cisplatin. The patient records between November, 2011 and January, 2013 were reviewed in our institution. A total of 5 patients had received EGFR-TKI and/or chemotherapy prior to developing BM and 4 of these patients had developed progressive disease (PD) after receiving $E G F R$-TKI. The study was approved by the Institutional Review Board of the First Affiliated Hospital of Guangzhou Medical University and written informed consent for genetic analyses was obtained from each patient at the time of diagnosis or operation.

Treatment and response evaluation. Combination therapy with erlotinib and pemetrexed/cisplatin was administered. Pemetrexed $\left(500 \mathrm{mg} / \mathrm{m}^{2}\right)$ and cisplatin $\left(20 \mathrm{mg} / \mathrm{m}^{2}\right)$ were administered on day 1 and days 1-3, respectively. Erlotinib $(150 \mathrm{mg}$ ) was administered daily on days $4-20$. The combination regimen was repeated every 21 days. Prior to pemetrexed treatment, the patients were treated with folic acid and vitamin B12. The patients received at least 1 cycle of chemotherapy. In case of tumor regression or stable disease (SD), no more than 6 cycles were administered. If the disease became stable after the combination therapy, the patients continued receiving erlotinib treatment as a maintenance therapy until eventual disease progression or the development of intolerable toxicities. The patients were followed up using brain magnetic resonance imaging (MRI) prior to the second cycle initially and after every 2 cycles thereafter. Image scanning for extracranial lesions was repeated every 2 cycles after the first treatment. The tumor responses were evaluated according to the Response Evaluation Criteria In Solid Tumors (RECIST) 1.1, using computed tomography or MRI (12). Treatment-related toxicity was evaluated according to the National Cancer Institution Common Terminology Criteria for Adverse Events 3.0.

EGFR mutation analysis. Genomic DNA was extracted from formalin-fixed, paraffin-embedded (FFPE) primary lung tumor tissues prior to treatment, using the QIAamp DNA FFPE Tissue kit (Qiagen, Hilden, Germany). EGFR mutations were detected using the commercially available AmoyDx ${ }^{\circledR}$ Human EGFR Gene 29 Mutations Fluorescence PCR Diagnosis kit (Amoy Diagnostics Co., Ltd, Xiamen, China). This EGFR kit detects 29 mutations in exons 18-21, including T790M, L858R, L861Q, S768I, G719S, G719A, G719C, three insertions in exon 20 and 19 deletions in exon 19.

Statistical analysis. Progression-free survival (PFS) was measured from the day of the first treatment of BM, until radiologically confirmed tumor progression or death. OS was

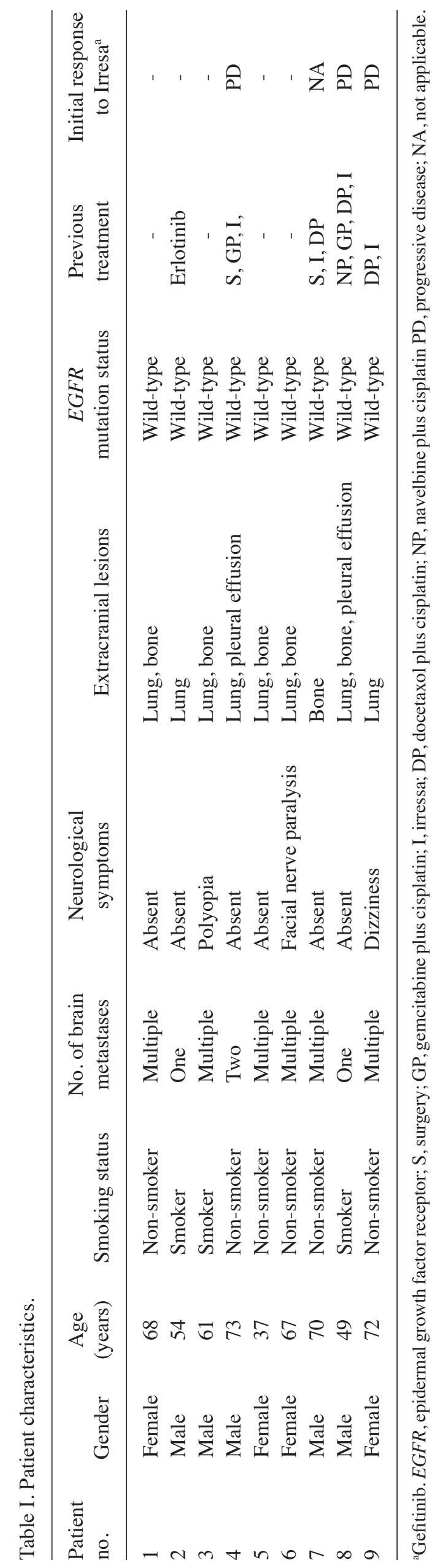


Table II. Tumor response and disease control.

\begin{tabular}{lccccccc}
\hline $\begin{array}{l}\text { Patient } \\
\text { no. }\end{array}$ & $\begin{array}{c}\text { Chemotherapy } \\
\text { cycles }\end{array}$ & $\begin{array}{c}\text { Intracranial } \\
\text { tumor } \\
\text { response }\end{array}$ & $\begin{array}{c}\text { Neurological } \\
\text { symptoms }\end{array}$ & $\begin{array}{c}\text { Extracranial } \\
\text { tumor } \\
\text { response }\end{array}$ & $\begin{array}{c}\text { Intracranial } \\
\text { disease control } \\
\text { (survival, days) }\end{array}$ & $\begin{array}{c}\text { Extracranial } \\
\text { disease control } \\
\text { (survival, days) }\end{array}$ & $\begin{array}{c}\text { Interval between therapy } \\
\text { and death or last } \\
\text { follow-up (days) }\end{array}$ \\
\hline 1 & 6 & PR & - & PR & 179 & 179 & 179 \\
2 & 5 & PR & - & PR & 206 & 114 & 236 \\
3 & 3 & PR & Improved & PD & 92 & 51 & 109 \\
4 & 3 & SD & - & PD & 107 & 59 & 107 \\
5 & 4 & PR & - & PR & 287 & 287 & 287 \\
6 & 1 & PR & Improved & SD & 144 & 201 & 232 \\
7 & 5 & PR & - & NA & 212 & - & 212 \\
8 & 1 & PD & - & PD & 23 & 23 & 141 \\
9 & 6 & PR & Improved & SD & 189 & 274 & 274 \\
\hline
\end{tabular}

PR, partial response; PD, progressive disease; SD, stable disease; NA, not-applicable. Patient no. 6 achieved a complete remission of the cranial tumor after cranial radiotherapy.
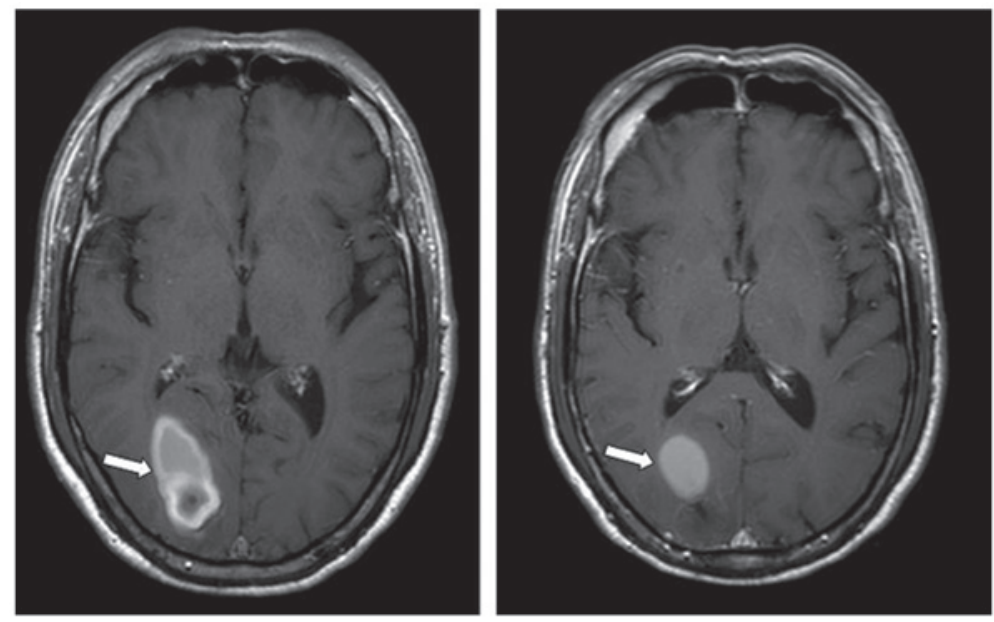

Figure 1. MRI imaging of brain metastasis. MRI imaging from a male patient (patient no. 3, Tables I and II). The cranial tumor exhibited a partial response (PR) after receiving one cycle of erlotinib plus pemetrexed/cisplatin. Left panel, prior to treatment. Right panel, image after one treatment cycle. MRI, magnetic resonance imaging.

measured from the first treatment of BM until death or the last follow-up. The last follow-up was in August, 2013.

\section{Results}

Patient characteristics. The patient characteristics are summarized in Table I. The 9 patients included 4 women and 5 men, aged 37-73 years at the time of diagnosis of BM. Six of the patients were non-smokers and 3 patients had neurological symptoms. The patients had received at least 1 platinum-based chemotherapy regimen. All the patients had extracranial disease progression and the emergence of BM, except patient 7 . We confirmed the presence of EGFR wild-type mutations in the primary tumor of all the patients, indicating that the $E G F R$ mutation was acquired during tumorigenesis.

Tumor response and disease control. All the patients received 1-6 cycles of chemotherapy (median, 4 cycles). The 3 patients with neurological symptoms exhibited an overt clinical response to the combination regimen for BM after the first cycle of treatment, with improvement of the metastasis-related neurological symptoms (Table II). According to RECIST, no patient with BM achieved a complete remission, 7 patients exhibited a partial response (PR), 1 had SD and 1 had PD. The objective response rate (ORR) was $77.8 \%$ (7/9). As regards the extracranial tumors, 3 patients exhibited a PR, 2 had SD, 3 had PD and 1 was not applicable (NA). The ORR was $37.5 \%(3 / 8)$. One patient's cranial MRI revealed tumor regression after treatment (Fig. 1).

At the last follow-up, 4 patients remained alive. The intracranial and extracranial median PFS were 179 and 146.5 days, respectively, and the median OS was 197.4 days (Fig. 2). The intracranial and extracranial disease of patient no. 5 has remained stable for 287 days after the first combination therapy and the patient remains alive to date.

Toxicity and side effects. A grade 1-2 rash developed in 7 patients, grade 2 oral mucositis in 1 patient and grade 2 


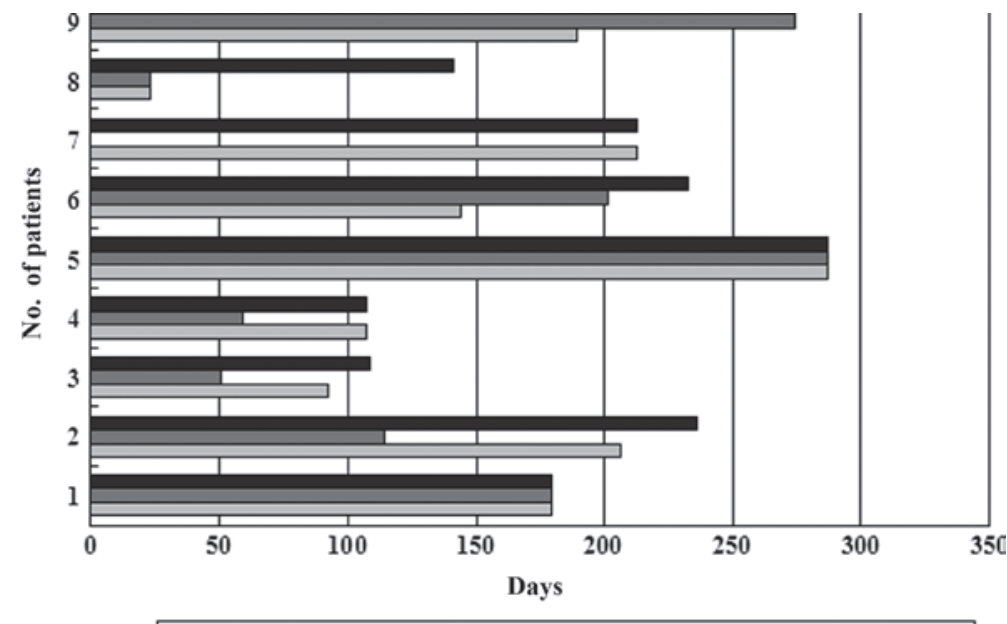

$\square$ Intracranial disease control $\square$ Extracranial disease control @Overall survival

Figure 2. Progression-free and overall survival of 9 patients by extracranial or intracranial disease control.

diarrhea in 1 patient. The combination regimen was relatively well-tolerated by the patients, with only grade 1-2 hematological toxicity.

\section{Discussion}

Approximately half of lung adenocarcinoma cases in Eastern Asia are encountered in patients harboring wild-type EGFR mutations and $46 \%$ of the lung adenocarcinoma patients reportedly respond to pemetrexed chemotherapy as first-line treatment (13-15). A Korean study on a small patient sample reported that non-smoking adenocarcinoma patients with asymptomatic BM achieved an ORR of $69.6 \%$ when treated with EGFR-TKI as first-line regimen (3). Another study reported an ORR of $58.3 \%$ in Chinese adenocarcinoma patients harboring $E G F R$-activating mutations with asymptomatic BM when treated with erlotinib as second-line therapy (16). EGFR wild-type patients exhibited significantly poorer BM treatment responses and shorter survival after BM diagnosis compared to EGFR-mutant patients (5).

The present study demonstrated that the intracranial treatment response was $77.8 \%$ (7/9). This may be due to the synergistic effects of erlotinib and pemetrexed, as supported by previous in vitro preclinical studies $(17,18)$. The TS inhibitor 5-fluorouracil may increase EGFR phosphorylation, thus potentially enhancing EGFR-TKI activity (19). Moreover, in vitro studies demonstrated that $E G F R$-TKIs may decrease TS expression and activity, undermining its synergistic interaction with 5'-deoxy-5-fluorouridine (20-21). Hence, erlotinib combined with pemetrexed may be a feasible and effective alternative for the treatment of lung adenocarcinoma concurrent with $\mathrm{BM}$ in the clinical setting.

There was a question whether the new regimen, erlotinib combined with pemetrexed and platinum, achieved superior responses in intracranial compared to extracranial disease. Our study demonstrated that the ORR of intracranial and extracranial treatment was 77.8 and $37.5 \%$, respectively. Moreover, we observed that intracranial disease control exhibited a trend to be superior to extracranial disease control (179 vs.
146.5 days, respectively). The patients tolerated the regimen well, albeit with the development of grade 1-2 rash, oral mucositis or diarrhea. The preliminary results from small patient samples appear to be promising; however, further large-scale studies are required to confirm these findings.

The results of this study may provide a new clinical application for the treatment of EGFR wild-type lung adenocarcinoma patients with BM. However, there are several limitations to our study. First, although a recent study reported that heterogeneous distribution of EGFR mutations is extremely rare in lung adenocarcinoma (22), the possibility of discrepancies between the EGFR mutation status of primary lung cancer and synchronous or metachronous BM could not be excluded and may affect our results. This limitation arises from the difficulties of performing additional biopsies of the BM in each patient. Second, the patient number in the present study was limited and not every patient was eligible for evaluation of the treatment response. Third, the imaging response may be miscalculated by RECIST, as this set of criteria has well-known limitations in the assessment of brain lesions, including only linear measurement of the tumor size, mere evaluation of target lesions $\geq 10 \mathrm{~mm}$ and lack of monitoring of biological tumor response (23). Moreover, the majority of the patients received more than one treatment modality for either BM or primary lung cancer after diagnosis of BM in our clinical practice, which may affect treatment response and survival.

In conclusion, erlotinib combined with pemetrexed/cisplatin was found to be effective and well-tolerated for the treatment of EGFR wild-type lung adenocarcinoma patients with BM. However, further clinical trials are required to confirm our results.

\section{References}

1. Hazard LJ, Jensen RL and Shrieve DC: Role of stereotactic radiosurgery in the treatment of brain metastases. Am J Clin Oncol 28: 403-410, 2005.

2. Chi A and Komaki R: Treatment of brain metastasis from lung cancer. Cancers (Basel) 2: 2100-2137, 2010. 
3. Kim JE, Lee DH, Choi Y, et al: Epidermal growth factor receptor tyrosine kinase inhibitors as a first-line therapy for never-smokers with adenocarcinoma of the lung having asymptomatic synchronous brain metastasis. Lung Cancer 65: 351-354, 2009.

4. Porta R, Sánchez-Torres JM, Paz-Ares L, et al: Brain metastases from lung cancer responding to erlotinib: the importance of EGFR mutation. Eur Respir J 37: 624-631, 2011.

5. Hsiao SH, Lin HC, Chou YT, Lin SE, Kuo CC, Yu MC and Chung CL: Impact of epidermal growth factor receptor mutations on intracranial treatment response and survival after brain metastases in lung adenocarcinoma patients. Lung Cancer 81: 455-461, 2013.

6. Shih C, Chen VJ, Gossett LS, et al: LY231514, a pyrrolo [2,3-d] pyrimidine-based antifolate that inhibits multiple folate-requiring enzymes. Cancer Res 57: 1116-1123, 1997.

7. Bailon O, Chouahnia K, Augier A, et al: Upfront association of carboplatin plus pemetrexed in patients with brain metastases of lung adenocarcinoma. Neuro Oncol 14: 491-495, 2012.

8. Sigmond J, Backus HH, Wouters D, Temmink OH, Jansen G and Peters GJ: Induction of resistance to the multitargeted antifolate pemetrexed (ALIMTA) in WiDr human colon cancer cells is associated with thymidylate synthase overexpression. Biochem Pharmacol 66: 431-438, 2003.

9. Spicer J and Harper P: Targeted therapies for non-small cell lung cancer. Int J Clin Pract 59: 1055-1062, 2005.

10. Ranson M, Reck M, Anthoney A, et al: Erlotinib in combination with pemetrexed for patients with advanced non-small-cell lung cancer (NSCLC): a phase I dose-finding study. Ann Oncol 21 : 2233-2239, 2010

11. Minami S, Kijima T, Takahashi R, et al: Combination chemotherapy with intermittent erlotinib and pemetrexed for pretreated patients with advanced non-small cell lung cancer: a phase I dose-finding study. BMC Cancer 12: 296, 2012.

12. Eisenhauer EA, Therasse P, Bogaerts J, et al: New response evaluation criteria in solid tumours: revised RECIST guideline (version 1.1). Eur J Cancer 45: 228-247, 2009.

13. Liang Z, Zhang J, Zeng X, et al: Relationship between EGFR expression, copy number and mutation in lung adenocarcinomas. BMC Cancer 10: 376, 2010.
14. Li C, Sun Y, Fang Z, et al: Comprehensive analysis of epidermal growth factor receptor gene status in lung adenocarcinoma. J Thorac Oncol 6: 1016-1021, 2011.

15. Orlando M, Lee JS, Yang C, Simms L and Park K: Efficacy of pemetrexed-cisplatin (PC) in East Asian patients (pts) Subgroup analysis of a phase III study comparing PC versus gemcitabine-cisplatin (GC) in first-line treatment of advanced nonsmall cell lung cancer (NSCLC). J Clin Oncol 27: 8045, 2009.

16. Wu YL,Zhou C, Cheng Y, et al: Erlotinib as second-line treatment in patients with advanced non-small-cell lung cancer and asymptomatic brain metastases: a phase II study (CTONG-0803). Ann Oncol 24: 993-999, 2013.

17. Giovannetti E, Lemos C, Tekle C, et al: Molecular mechanisms underlying the synergistic interaction of erlotinib, an epidermal growth factor receptor tyrosine kinase inhibitor, with the multitargeted antifolate pemetrexed in non-small-cell lung cancer cells. Mol Pharmacol 73: 1290-1300, 2008.

18. Li T, Ling YH, Goldman ID and Perez-Soler R: Schedule-dependent cytotoxic synergism of pemetrexed and erlotinib in human non-small cell lung cancer cells. Clin Cancer Res 13: 3413-3422, 2007.

19. Van Schaeybroeck S, Karaiskou-McCaul A, Kelly D, Longley D, Galligan L, VanCutsem E and Johnston P: Epidermal growth factor receptor activity determines response of colorectal cancer cells to gefitinib alone and in combination with chemotherapy. Clin Cancer Res 11: 7480-7489, 2005.

20. Magne N, Fischel JL, Dubreuil A, et al: ZD1839 (Iressa) modifies the activity of key enzymes linked to fluoropyrimidine activity: rational basis for a new combination therapy with capecitabine. Clin Cancer Res 9: 4735-4742, 2003.

21. Budman DR, Soong R, Calabro A, Tai J and Diasio R: Identification of potentially useful combinations of epidermal growth factor receptor tyrosine kinase antagonists with conventional cytotoxic agents using median effect analysis. Anticancer Drugs 17: 921-928, 2006

22. Yatabe Y,Matsuo K and Mitsudomi T: Heterogeneous distribution of EGFR mutations is extremely rare in lung adenocarcinoma. J Clin Oncol 29: 2972-2977, 2011.

23. Henson JW, Ulmer S and Harris GJ: Brain tumor imaging in clinical trials. AJNR Am J Neuroradiol 29: 419-424, 2008. 